\title{
Computational examples of rational string operations on Gorenstein spaces
}

\author{
Takahito Naito
}

\begin{abstract}
In this paper, we give computational examples of string operations over the rational numbers field on Gorenstein spaces introduced by Félix and Thomas. Especially, we determine the structure of rational string operations on the classifying space of a compact connected Lie group and the Borel construction associated to an action of $S^{1}$ to $S^{2}$.
\end{abstract}

\section{Introduction}

Chas and Sullivan [1] discovered an intersection-type product on the homology of the free loop space of manifold $H_{*}(L M)$ called the loop product. This algebraic structure has been computed by several authors [3, 7, 12,9]. Cohen and Godin [2] generalized the loop product and they showed that $H_{*}(L M)$ is a 2-dimensional TQFT. Especially, we can see that the homology is endowed with a coproduct which is called the loop coproduct. However, Tamanoi [11] proved that the loop coproduct is almost trivial.

Félix and Thomas [6] developed string topology to Gorenstein spaces. For example, closed oriented manifolds, the classifying spaces of compact connected Lie groups and the Borel constructions are Gorenstein spaces. They introduced the product and the coproduct on the homology of the free loop spaces of simplyconnected Gorenstein spaces which are generalizations of the loop product and coproduct. However, there are few computational examples of the algebraic structures on Gorenstein spaces which are not manifolds. The aim of this paper

Received by the editors in September 2014 - In revised form in January 2015.

Communicated by Y. Félix.

2010 Mathematics Subject Classification : Primary 55P50; Secondary 55P62.

Key words and phrases : string topology, Gorenstein space, rational homotopy theory. 
is to give some computational examples of the loop (co)product over the rational numbers field $\mathbb{Q}$ on Gorenstein spaces by using minimal Sullivan models.

In this paper, we compute the rational loop (co)product of the classifying space of a compact connected Lie group $B G$ and a Borel construction $E S^{1} \times_{S^{1}} S^{2}$ associated to an action of $S^{1}$ to $S^{2}=\left\{(z, r) \in \mathbb{C} \times\left.\mathbb{R}|| z\right|^{2}+r^{2}=1\right\}$ given by $t \cdot(z, r)=(t z, r)$ for $t \in S^{1}$. By the result [6] due to Félix and Thomas, the rational loop product on $B G$ vanishes. In [8], Kuribayashi and Menichi also computed the loop coproduct of $B G$. We claim that the computational approach to the loop coproduct in this paper are different from theirs. Complete calculations of the rational loop (co)product of $B G$ and $E S^{1} \times{ }_{S^{1}} S^{2}$ are stated in $\S 3$ and $\S 4$, respectively.

As mentioned above, either the loop product or the loop coproduct of manifolds and the classifying spaces are almost trivial. However, we can see that the algebraic structures of general Gorenstein spaces are non-trivial by the computations of the loop (co)product of $E S^{1} \times{ }_{S^{1}} S^{2}$.

The organization of this paper is as follows. In section 2 , we recall fundamental definitions and results on rational homotopy theory. The definition of the dual loop (co) product on Gorenstein space and its Sullivan models are also described. In section 3, we will give a computation of the dual loop coproduct on $H^{*}(L B G ; \mathbb{Q})$. Computations of the homology $H^{*}\left(L\left(E S^{1} \times_{S^{1}} S^{2}\right) ; \mathbb{Q}\right)$ and the dual loop (co)product are presented in $\S 4$.

\section{Preliminaries}

In this paper, we assume that all modules and algebras are over $\mathbb{Q}$.

\subsection{Minimal Sullivan models and a model for the free loop space}

We refer the reader to the book [5] for the fundamental facts on rational homotopy theory. In this section, we begin recalling the definition of a minimal Sullivan model for a simply-connected space $X$ with finite Betti numbers. It is a free commutative differential graded algebra (dg algebra for short) $(\Lambda V, d)$ with $V=\oplus_{i \geq 2} V^{i}$ where each $V^{i}$ is of finite dimension and the differential $d$ is decomposable; that is, $d(V) \subset \Lambda^{\geq 2} V$. Moreover, $(\Lambda V, d)$ is equipped with a quasiisomorphism $(\Lambda V, d) \longrightarrow A_{\mathrm{PL}}(X)$ to the commutative differential graded algebra $A_{\mathrm{PL}}(X)$ of differential polynomial forms on $X$.

Let $(\Lambda V, d)$ be a minimal Sullivan model for $X$. Denote by $s V$ the suspension of $V$; that is, $s V$ is a graded vector space defined by $(s V)^{i}=V^{i+1}$, and by $\tilde{v} \in s V$ the element which corresponds to $v \in V$. We then define a commutative dg algebra $\Lambda V \otimes \Lambda V \otimes \Lambda(s V)$ with a differential $D$ given by

$$
\begin{aligned}
& D(v \otimes 1 \otimes 1)=d(v) \otimes 1 \otimes 1, D(1 \otimes v \otimes 1)=1 \otimes d(v) \otimes 1, \\
& D(1 \otimes 1 \otimes \tilde{v})=-v \otimes 1 \otimes 1+1 \otimes v \otimes 1-\sum_{i=1}^{\infty} \frac{(s D)^{i}}{i !}(v \otimes 1 \otimes 1)
\end{aligned}
$$

where $s$ is the unique derivation on $\Lambda V \otimes \Lambda V \otimes \Lambda(s V)$ defined by

$$
s(v \otimes 1 \otimes 1)=s(1 \otimes v \otimes 1)=1 \otimes 1 \otimes \tilde{v}, \quad s(1 \otimes 1 \otimes \tilde{v})=0 .
$$


Lemma 2.1. The map

$$
\bar{\varepsilon}=\mu \cdot \varepsilon:(\Lambda V \otimes \Lambda V \otimes \Lambda(s V), D) \longrightarrow(\Lambda V, d)
$$

is a right $\Lambda V \otimes \Lambda V$-semifree resolution of $\Lambda V$. Here $\mu$ is the product on $\Lambda V$ and $\varepsilon: \Lambda(s V) \rightarrow \mathbb{Q}$ is the canonical augmentation.

Remark that the dg algebra $(\Lambda V \otimes \Lambda V \otimes \Lambda(s V), D)$ is a model for the path space $X^{I}=\operatorname{Map}([0,1], X)$ and the map $\bar{\varepsilon}$ is a model for $c: X \rightarrow X^{I}$ which sends $x$ to the constant path at $x$.

By $[5, \S 15$ Example 1], the dg algebra

$$
(\Lambda V, d) \otimes_{\Lambda V \otimes \Lambda V}(\Lambda V \otimes \Lambda V \otimes \Lambda(s V), D) \cong(\Lambda V \otimes \Lambda(s V), \bar{D})
$$

is a model for the free loop space $L X$, namely, $H^{*}(\Lambda V \otimes \Lambda(s V), \bar{D})$ is isomorphic to $H^{*}(L X ; \mathbb{Q})$ as an algebra. The induced differential $\bar{D}$ is given by

$$
\bar{D}(v \otimes 1)=d(v) \otimes 1, \quad \bar{D}(1 \otimes \tilde{v})=-s(1 \otimes d(v))
$$

for $v \in V$. In this paper, we denote by

$$
\mathcal{M}_{X^{I}}:=(\Lambda V \otimes \Lambda V \otimes \Lambda(s V), D), \quad \mathcal{M}_{L X}:=(\Lambda V \otimes \Lambda(s V), \bar{D})
$$

for short. By the result [13] due to Vigué, $\mathcal{M}_{L X}$ decomposes into a direct sum of complexes as follows

$$
\mathcal{M}_{L X} \cong \bigoplus_{i=0}^{\infty}\left(\Lambda V \otimes \Lambda^{i}(s V), \bar{D}\right)
$$

and we denote by $H_{(i)}^{*}(L X ; \mathbb{Q})$ the homology of the $i$-th direct summand $\left(\Lambda V \otimes \Lambda^{i}(s V), \bar{D}\right)$. By the definition, the homology $H_{(0)}^{*}(L X ; \mathbb{Q})$ is isomorphic to $H^{*}(\Lambda V, d) \cong H^{*}(X ; \mathbb{Q})$ as an algebra.

\subsection{The loop (co)product on Gorenstein spaces and its models}

We recall the definition of Gorenstein spaces and the dual loop product and coproduct on Gorenstein spaces. Let $C^{*}(-)$ be the normalized singular cochain algebra functor over $\mathbb{Q}$.

Definition $2.2([4])$. A connected space $M$ is a $\mathbf{Q}$-Gorenstein space of dimension $m$ if

$$
\operatorname{Ext}_{C^{*}(M)}^{*}\left(\mathbb{Q}, C^{*}(M)\right)= \begin{cases}0 & (* \neq m) \\ \mathbb{Q} & (*=m) .\end{cases}
$$

For example, a closed oriented manifold $M$ is a Gorenstein space. The dimension of $M$ as Gorenstein space coincides with the dimension as manifold. The classifying space $B G$ of a compact connected Lie group is also a Gorenstein space, and the dimension of $B G$ is - $\operatorname{dim} G$. Félix, Halperin and Thomas proved that a simply-connected space $X$ with $\sum \operatorname{dim} \pi_{i}(X) \otimes \mathbb{Q}<\infty$ is a $\mathbb{Q}$-Gorenstein 
space ([4, Proposition 3.4]).

Let $M$ be a simply-connected $m$-dimensional $Q$-Gorenstein space of finite type. In [6], Félix and Thomas proved that

$$
\operatorname{Ext}_{C^{*}\left(M^{2}\right)}^{m}\left(C^{*}(M), C^{*}\left(M^{2}\right)\right) \cong \mathbb{Q}
$$

as a vector space where $C^{*}(M)$ is a $C^{*}\left(M^{2}\right)$-module via the diagonal map of $M$. Let $P \rightarrow C^{*}(M)$ be a right $C^{*}\left(M^{2}\right)$-semifree resolution of $C^{*}(M)$ and denote by $\Delta^{!}: P \rightarrow C^{*}\left(M^{2}\right)$ the element of $\operatorname{Ext}_{C^{*}\left(M^{2}\right)}^{m}\left(C^{*}(M), C^{*}\left(M^{2}\right)\right)$ which corresponds to $1 \in \mathbb{Q}$. We remark that if $M$ is a simply-connected closed smooth manifold, the induced map $H\left(\Delta^{!}\right): H^{*}(M) \cong H^{*}(P) \rightarrow H^{*}(M)^{\otimes 2}$ is the dual of the intersection product.

Let $L M \times{ }_{M} L M$ denote the subspace of the product $L M \times L M$ consisting of the pairs of loops having the same basepoint, and comp : $L M \times_{M} L M \rightarrow L M$ the concatenation of loops. Consider the diagram

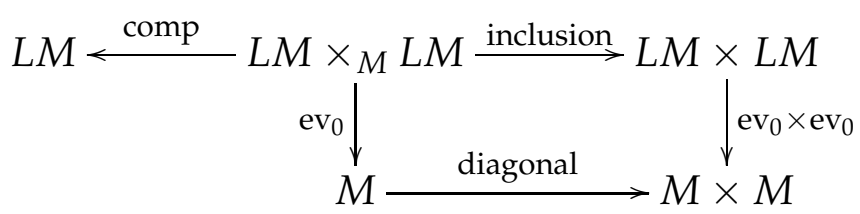

where $\mathrm{ev}_{0}$ is the evaluation map at 0 . Since the right square is a pullback diagram, there is an isomorphism $\mathrm{EM}_{1}: H^{*}\left(P \otimes_{C^{*}\left(M^{2}\right)} C^{*}(L M \times L M)\right) \rightarrow H^{*}\left(L M \times_{M} L M\right)$ associated to the pullback diagram which is called the Eilenberg-Moore isomorphism. For details of the Eilenberg-Moore map, see [10]. Then, we call the following composite the dual loop product:

$$
\operatorname{Dlp}: H^{*}(L M) \stackrel{\text { comp }^{*}}{\longrightarrow} H^{*}\left(L M \times_{M} L M\right) \stackrel{\left(\Delta^{!} \otimes 1\right) \mathrm{EM}_{1}^{-1}}{\longrightarrow} H^{*}(L M \times L M) .
$$

Consider the diagram

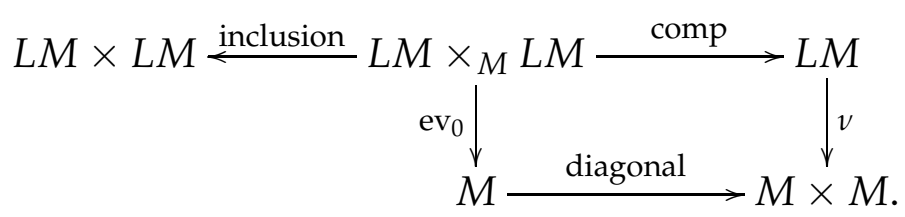

Here the map $v$ sends a loop $\gamma \in L M$ to $\left(\gamma(0), \gamma\left(\frac{1}{2}\right)\right)$. Denote by $\mathrm{EM}_{2}: H^{*}\left(P \otimes_{C^{*}\left(M^{2}\right)} C^{*}(L M)\right) \rightarrow H^{*}\left(L M \times_{M} L M\right)$ the Eilenberg-Moore map associated to the pullback. Then we call the following composite the dual loop coproduct:

Dlcop : $H^{*}(L M \times L M) \stackrel{\text { (inclusion) }}{\longrightarrow} H^{*}\left(L M \times \times_{M} L M\right) \stackrel{\left(\Delta^{!} \otimes 1\right) \mathrm{EM}_{2}^{-1}}{\longrightarrow} H^{*}(L M)$.

We next mention about a commutativity of the dual loop coproduct for a computation of the dual loop coproduct on $H^{*}\left(L\left(E S^{1} \times_{S^{1}} S^{2}\right) ; \mathbb{Q}\right)$ in $\S 4$. Let $t: M^{2} \rightarrow M^{2}$ be the switching map. Denote by $P^{\prime}$ the right $C^{*}\left(M^{2}\right)$-module which is equal as vector space to $P$ with a right $C^{*}\left(M^{2}\right)$-module structure given 
by the algebra map $t^{*}: C^{*}\left(M^{2}\right) \rightarrow C^{*}\left(M^{2}\right)$. The module $P^{\prime}$ is also a semifree resolution of $C^{*}(M)$ as right $C^{*}\left(M^{2}\right)$-module. Let $\bar{T}: P \rightarrow P^{\prime}$ is a $C^{*}\left(M^{2}\right)$-module map which makes the following diagram homotopy commutative:

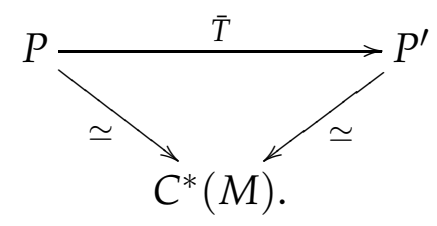

A existence of $\bar{T}$ is shown by a property of semifree modules; see [5, Proposition 6.4].

Consider the composite

$$
P \stackrel{\bar{T}}{\longrightarrow} P^{\prime} \stackrel{\Delta^{!}}{\longrightarrow} C^{*}\left(M^{2}\right) \stackrel{t^{*}}{\longrightarrow} C^{*}\left(M^{2}\right) .
$$

Since $\Delta^{!}$and $t^{*} \circ \Delta^{!} \circ \bar{T}$ are elements in $\operatorname{Ext}_{C^{*}\left(M^{2}\right)}^{m}\left(C^{*}(M), C^{*}\left(M^{2}\right)\right) \cong \mathbb{Q}$, there exists a scalar $\lambda \in \mathbb{Q}$ which makes the diagram of $C^{*}\left(M^{2}\right)$-modules commute up to homotopy:

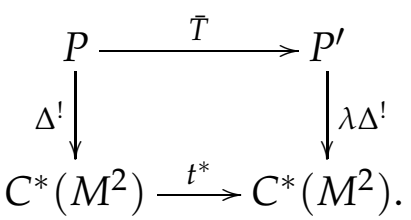

We then have the following lemma.

Lemma 2.3. Let $\lambda$ be the scalar described above. Then Dlcop $\circ \tilde{t}^{*}=\lambda \mathrm{Dlcop}$, where $\tilde{t}:(L M)^{2} \rightarrow(L M)^{2}$ is the switching map of $L M$.

Proof. Let $R_{\frac{1}{2}}: L M \rightarrow L M$ be the rotation of loops by $\frac{1}{2}$. Consider the commutative diagram

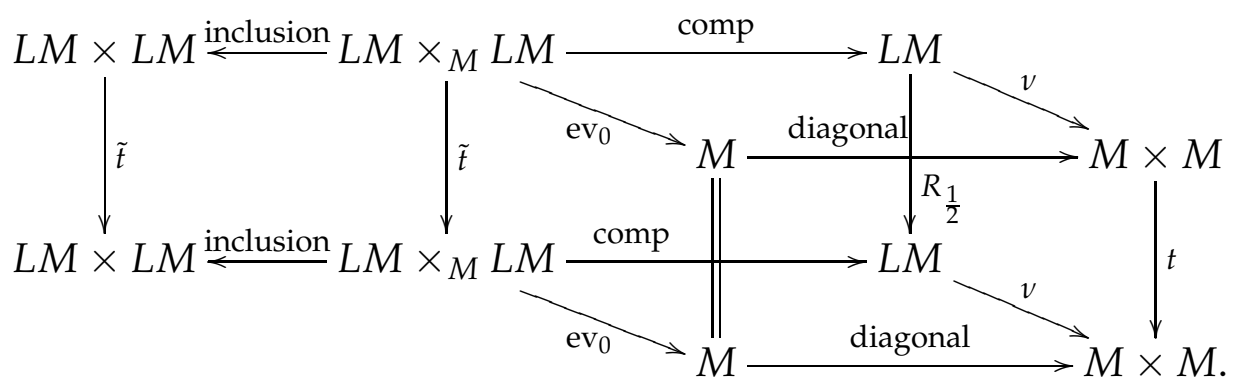

The assumption and a naturality of the Eilenberg-Moore map show that the following is commutative:

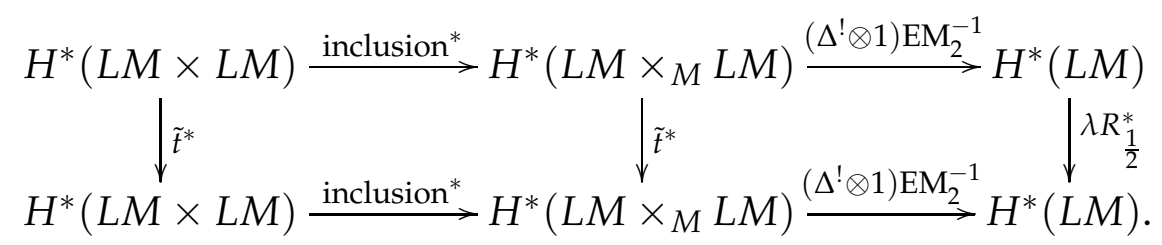

Since $R_{\frac{1}{2}}$ is homotopic to the identity map of $L M$, it follows the assertion. 
In the rest of this section, we describe models for the dual loop product and coproduct. Consider the following diagram whose top and bottom squares are pullbacks:

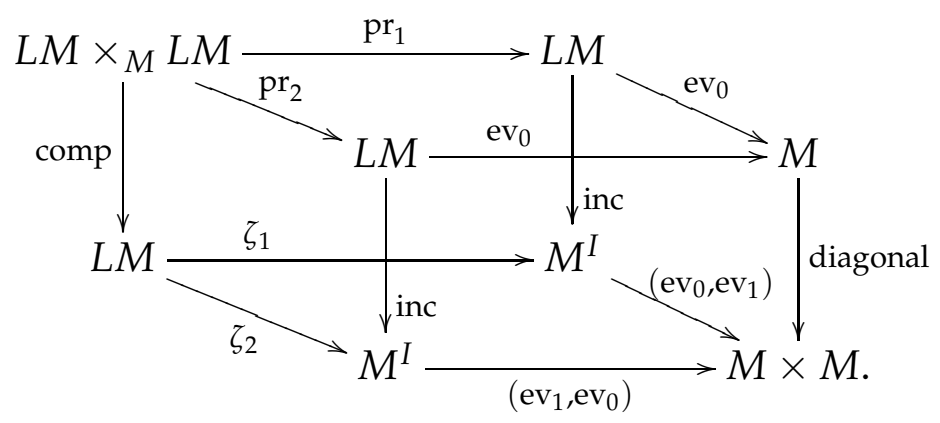

Here inc $: L M \rightarrow M^{I}$ is the inclusion, $\mathrm{ev}_{i}: M^{I} \rightarrow M$ is the evaluation map at $i$, and $\mathrm{pr}_{i}$ is the $i$-th projection. The map $\zeta_{1}$ and $\zeta_{2}$ are given by $\zeta_{1}(\gamma)(t)=\gamma\left(\frac{1}{2} t\right)$, $\zeta_{2}(\gamma)(t)=\gamma\left(\frac{1}{2} t+\frac{1}{2}\right)$ for $\gamma \in L M$ and $t \in[0,1]$. This diagram enables us to obtain a model for the map comp as follows:

$$
\mathcal{M}_{L M} \stackrel{\bar{\varepsilon} \otimes 1}{\simeq} \mathcal{M}_{M^{I}} \otimes_{\Lambda V^{\otimes 2}} \mathcal{M}_{M^{I}}^{(\mu \otimes 1) \otimes_{\mu}(\mu \otimes 1)} \mathcal{M}_{L M} \otimes_{\Lambda V} \mathcal{M}_{L M}
$$

Remark that the left $\Lambda V^{\otimes 2}$-module structure of $\mathcal{M}_{M^{I}}$ is given by an algebra map $\Lambda V^{\otimes 2} \rightarrow \mathcal{M}_{M^{I}}$ which sends $a \otimes b$ to $(-1)^{|a||b|} b \otimes a \otimes 1$ where the notion $|a|$ is the degree of $a$. This algebra map is a model for $\left(\mathrm{ev}_{1}, \mathrm{ev}_{0}\right): M^{I} \rightarrow M^{2}$. Therefore, the following is a model for the dual loop product, namely, the induced map in homology coincides with Dlp:

$$
\begin{gathered}
\mathcal{M}_{L M} \stackrel{\bar{\varepsilon} \otimes 1}{\simeq} \mathcal{M}_{M^{I}} \otimes_{\Lambda V^{\otimes 2}} \mathcal{M}_{M^{I}} \stackrel{(\mu \otimes 1) \otimes_{\mu}(\mu \otimes 1)}{\longrightarrow} \mathcal{M}_{L M} \otimes_{\Lambda V} \mathcal{M}_{L M} \\
\mathcal{M}_{L M}^{\otimes 2} \stackrel{\mid \cong}{\stackrel{\Delta^{!} \otimes 1}{\longleftarrow}} \mathcal{M}_{M^{I}} \otimes_{\Lambda V \otimes 2}\left(\mathcal{M}_{L M}^{\otimes 2}\right) \stackrel{\bar{\varepsilon} \otimes 1}{\simeq} \Lambda V \otimes_{\Lambda V} \underset{\sim}{\simeq}\left(\mathcal{M}_{L M}^{\otimes 2}\right) .
\end{gathered}
$$

A model for the dual loop coproduct is obtained as follows. Consider the two commutative diagrams

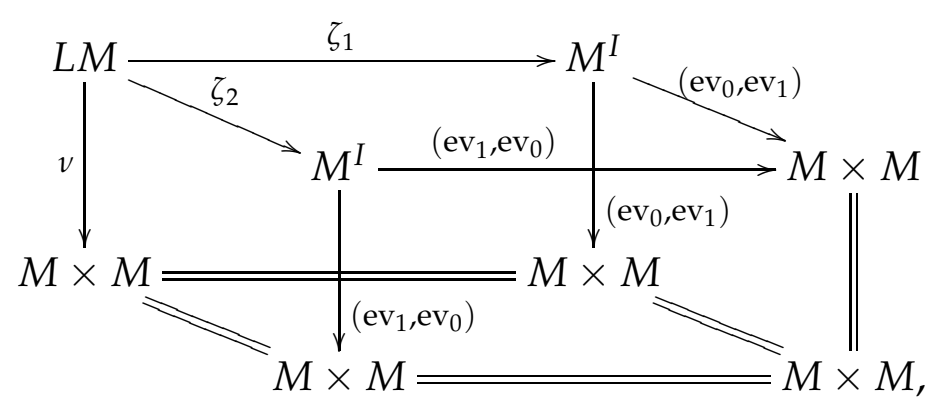




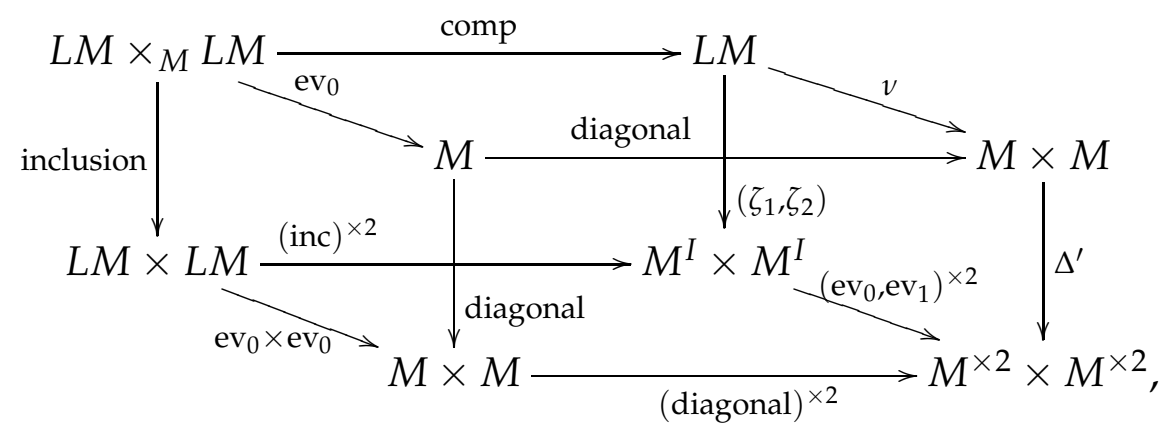

where $\Delta^{\prime}$ is a map which sends $(x, y)$ to $(x, y, y, x)$. We notice that each top and bottom squares are pullback diagrams. By the diagram (3), the inclusion $\Lambda V^{\otimes 2} \rightarrow \mathcal{M}_{M^{I}} \otimes_{\Lambda V^{\otimes 2}} \mathcal{M}_{M^{I}}$ is a model for $v$. Since the quotient map

$$
\bar{\zeta}: \mathcal{M}_{M^{I}} \otimes \mathcal{M}_{M^{I}} \longrightarrow \mathcal{M}_{M^{I}} \otimes_{\Lambda V^{\otimes 2}} \mathcal{M}_{M^{I}}
$$

is a model for $\left(\zeta_{1}, \zeta_{2}\right)$, by the diagram (4), the following composite is a model for the inclusion $L M \times{ }_{M} L M \rightarrow L M \times L M$ :

$$
\mathcal{M}_{L M}^{\otimes 2} \cong \Lambda V^{\otimes 2} \otimes_{\Lambda V^{\otimes 4}} \mathcal{M}_{M^{I}}^{\otimes 2} \stackrel{\mu \otimes_{\mu^{\prime}} \bar{\xi}}{\longrightarrow} \Lambda V \otimes_{\Lambda V^{\otimes 2}}\left(\mathcal{M}_{M^{I}} \otimes_{\Lambda V^{\otimes 2}} \mathcal{M}_{M^{I}}\right),
$$

where $\mu^{\prime}: \Lambda V^{\otimes 4} \rightarrow \Lambda V^{\otimes 2}$ is a model for $\Delta^{\prime}$ given by $\mu^{\prime}\left(v_{1} \otimes v_{2} \otimes v_{3} \otimes v_{4}\right)=$ $(-1)^{\left|v_{4}\right|\left(\left|v_{2}\right|+\left|v_{3}\right|\right)} v_{1} v_{4} \otimes v_{2} v_{3}$. Thus a map induced by the following in homology is the dual loop coproduct:

$$
\begin{array}{r}
\mathcal{M}_{L M}^{\otimes 2} \stackrel{\mu \otimes_{\mu^{\prime}} \bar{\xi}}{\longrightarrow} \Lambda V \otimes_{\Lambda V^{\otimes 2}}\left(\mathcal{M}_{M^{I}} \otimes_{\Lambda V^{\otimes 2}} \mathcal{M}_{M^{I}}\right) \\
\simeq \uparrow_{\bar{\varepsilon} \otimes 1} \\
\mathcal{M}_{L M} \stackrel{\bar{\varepsilon} \otimes 1}{\simeq} \mathcal{M}_{M^{I}} \otimes_{\Lambda V^{\otimes 2}} \mathcal{M}_{M^{I}} \stackrel{\Delta^{\prime} \otimes 1}{\longleftarrow} \mathcal{M}_{M^{I}} \otimes_{\Lambda V^{\otimes 2}}\left(\mathcal{M}_{M^{I}} \otimes_{\Lambda V^{\otimes 2}} \mathcal{M}_{M^{I}}\right) .
\end{array}
$$

Let $T: \Lambda V^{\otimes 2} \rightarrow \Lambda V^{\otimes 2}$ be the switching map and denote by $\bar{T}$ a map which makes the following diagram commutative:

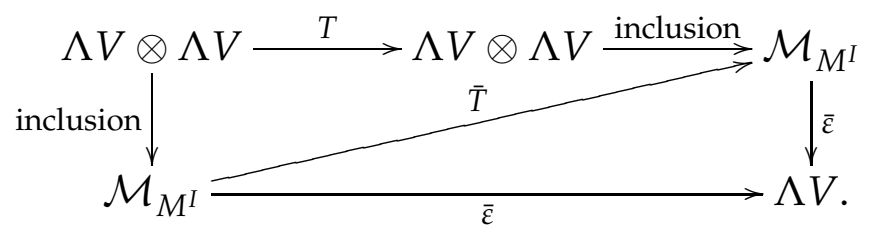

Remark that $\bar{T}$ coincides with the map stated in Lemma 6 up to homotopy. Then we note that the following diagram is commutative

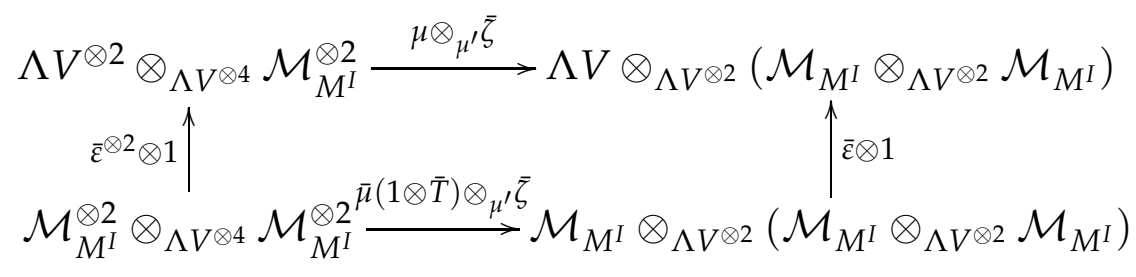


where $\bar{\mu}$ is the product on $\mathcal{M}_{M^{I}}$. By combining the diagrams (5) and (7), we have a model for the dual loop coproduct as follows:

$$
\begin{gathered}
\mathcal{M}_{L M}^{\otimes 2} \stackrel{\bar{\varepsilon}^{\otimes 2} \otimes 1}{\simeq} \mathcal{M}_{M^{I}}^{\otimes 2} \otimes_{\Lambda V^{\otimes 4}} \mathcal{M}_{M^{I}}^{\otimes 2} \\
\mathcal{M}_{L M} \stackrel{\bar{\varepsilon} \otimes 1}{\simeq} \mathcal{M}_{M^{I}} \otimes_{\Lambda V^{\otimes 2}} \mathcal{M}_{M^{I}} \stackrel{\Delta^{!} \otimes 1}{\longleftarrow} \mathcal{M}_{M^{I}} \otimes_{\Lambda V^{\otimes 2}}\left(\mathcal{M}_{M^{I}} \otimes_{\Lambda V^{\otimes 2}} \mathcal{M}_{M^{I}}\right) .
\end{gathered}
$$

\section{The dual loop coproduct of classifying spaces}

In this section, we observe the rational dual loop (co)product on the classifying space $B G$ of a compact connected Lie group $G$. Recall that the rational cohomology ring of $B G$ is a polynomial algebra of the form $H^{*}(B G ; \mathbb{Q}) \cong \mathbb{Q}\left[x_{1}, \cdots, x_{n}\right]$, and $\mathbb{Q}\left[x_{1}, \cdots, x_{n}\right]$ with zero differential is a minimal Sullivan model for $B G$. We put $\mathcal{M}_{B G}=\mathbb{Q}\left[x_{1}, \cdots, x_{n}\right]$ for convenience. We also recall that $B G$ is a $\mathbb{Q}$-Gorenstein space of dimension $-\operatorname{dim} G=-\sum_{i}\left(\left|x_{i}\right|-1\right)$; see [4, Theorem 4.3].

We first determine a $\mathbb{Q}$-vector space structure of $H^{*}(L B G ; \mathbb{Q})$ for a calculation of the dual loop (co)product.

Lemma 3.1. The rational cohomology ring $H^{*}(L B G ; \mathbb{Q})$ is isomorphic to $\mathbb{Q}\left[x_{1}, \cdots, x_{n}\right] \otimes \Lambda\left(\tilde{x}_{1}, \cdots, \tilde{x}_{n}\right)$ with $\left|\tilde{x}_{i}\right|=\left|x_{i}\right|-1$.

Proof. Notice that the differential $\bar{D}$ of $\mathcal{M}_{L B G}$ stated in $\S 2.1$ is zero. It follows that $H^{*}(L B G ; \mathbb{Q}) \cong H^{*}\left(\mathcal{M}_{L B G}\right) \cong \mathbb{Q}\left[x_{1}, \cdots, x_{n}\right] \otimes \Lambda\left(\tilde{x}_{1}, \cdots, \tilde{x}_{n}\right)$.

Next, we calculate the dual loop (co)product on $H^{*}(L B G ; \mathbb{Q})$. As for the dual loop product, Félix and Thomas proved the following theorem.

Theorem 3.2 ([6, Theorem 14]). The rational dual loop product on $H^{*}(L B G ; \mathbb{Q})$ is trivial.

We determine the structure of the dual loop coproduct Dlcop on $H^{*}(L B G ; \mathbb{Q})$ as follows. Let $\mathcal{M}_{B G^{I}}=\left(\mathbb{Q}\left[x_{1}, \cdots, x_{n}\right]^{\otimes 2} \otimes \Lambda\left(\tilde{x}_{1}, \cdots, \tilde{x}_{n}\right), D\right)$ be the semifree resolution of $\mathcal{M}_{B G}$ stated in $\S 2.1$, and denote by $[n]=\{1,2, \cdots, n\}$. Define a $\mathcal{M}_{B G}^{\otimes 2}$-module map $\Delta^{!}: \mathcal{M}_{B G^{I}} \rightarrow \mathcal{M}_{B G}^{\otimes 2}$ by

$$
\Delta^{!}\left(\tilde{x}_{i_{1}} \tilde{x}_{i_{2}} \cdots \tilde{x}_{i_{k}}\right)= \begin{cases}1 & \left(k=n,\left\{i_{1}, \cdots, i_{k}\right\}=[n]\right), \\ 0 & \text { (otherwise). }\end{cases}
$$

It is readily seen that the map $\Delta !$ is a generator of $\operatorname{Ext}_{\mathcal{M}_{B G}^{\otimes 2}}^{-\operatorname{dim}^{\otimes}}\left(\mathcal{M}_{B G}, \mathcal{M}_{B G}^{\otimes 2}\right)$; also see $[6, \S 6]$. For any subset $I=\left\{i_{1}<i_{2}<\cdots<i_{k}\right\}$ of $[n]$, we put $\tilde{x}_{(I)}=\tilde{x}_{i_{1}} \tilde{x}_{i_{2}} \cdots \tilde{x}_{i_{k}}$ for convenience. If $I$ is the empty set $\varnothing$, put $\tilde{x}_{\varnothing}=1$.

Let us define a map $\bar{T}: \mathcal{M}_{B G^{I}} \rightarrow \mathcal{M}_{B G^{I}}$ by $\bar{T}(a \otimes b \otimes 1)=(-1)^{|a||b|} b \otimes a \otimes 1$ and $\bar{T}\left(\tilde{x}_{i}\right)=-\tilde{x}_{i}$ for $a, b \in \mathcal{M}_{B G}$. Note that this is a map mentioned in the diagram (6). 
Theorem 3.3. The dual loop coproduct on $H^{*}(L B G ; \mathbb{Q})$ satisfies

$$
\operatorname{Dlcop}\left(\left(\omega_{1} \otimes \tilde{x}_{(I)}\right) \otimes\left(\omega_{2} \otimes \tilde{x}_{(J)}\right)\right)=\left\{\begin{array}{cc}
(-1)^{\epsilon} \omega_{1} \omega_{2} \otimes \tilde{x}_{(I \cap J)} & (I \cup J=[n]), \\
0 & \text { (otherwise). }
\end{array}\right.
$$

Here, $\omega_{1}, \omega_{2} \in \mathbb{Q}\left[x_{1}, \cdots, x_{n}\right], \epsilon=\epsilon_{1}+\epsilon_{2}+\#(J-I \cap J), \epsilon_{1}$ and $\epsilon_{2}$ are the Koszul sign convention satisfying the following

$$
\tilde{x}_{(J)}=(-1)^{\epsilon_{1}} \tilde{x}_{(J-I \cap J)} \tilde{x}_{(I \cap J)}, \quad \tilde{x}_{([n])}=(-1)^{\epsilon_{2}} \tilde{x}_{(I)} \tilde{x}_{(J-I \cap J)} .
$$

Proof. Recall the model of the dual loop coproduct described in (8). The element $u=\left(1 \otimes 1 \otimes \tilde{x}_{i}\right) \otimes 1+1 \otimes\left(1 \otimes 1 \otimes \tilde{x}_{i}\right)$ in $\mathcal{M}_{B G^{I}} \otimes_{\mathcal{M}_{B G}^{\otimes 2}} \mathcal{M}_{B G^{I}}$ is a cycle satisfying $(\bar{\varepsilon} \otimes 1)(u)=\tilde{x}_{i}$. It follows that

$$
\begin{aligned}
& \left(1 \otimes \omega_{i}\right) \cdot \prod_{i \in I}\left(\left(1 \otimes 1 \otimes \tilde{x}_{i}\right) \otimes 1+1 \otimes\left(1 \otimes 1 \otimes \tilde{x}_{i}\right)\right) \\
= & \sum_{I^{\prime} \subset I}(-1)^{\epsilon\left(I: I^{\prime}\right)}\left(1 \otimes \omega_{1} \otimes \tilde{x}_{\left(I^{\prime}\right)}\right) \otimes \tilde{x}_{\left(I-I^{\prime}\right)}
\end{aligned}
$$

is also a cycle in $\mathcal{M}_{B G^{I}} \otimes_{\mathcal{M}_{B G}^{\otimes 2}} \mathcal{M}_{B G^{I}}$ such that $\bar{\varepsilon} \otimes 1$ sends this cycle to $\omega_{i} \otimes \tilde{x}_{(I)}$ in $\mathcal{M}_{L B G}$. Here $\epsilon\left(I: I^{\prime}\right)$ is the Koszul sing convention satisfying $\tilde{x}_{(I)}=(-1)^{\epsilon\left(I: I^{\prime}\right)} \tilde{x}_{\left(I^{\prime}\right)} \tilde{x}_{\left(I-I^{\prime}\right)}$.

Let $\theta$ be an element in $\mathcal{M}_{B G^{I}}^{\otimes 2} \otimes_{\mathcal{M}_{B G}^{\otimes 4}} \mathcal{M}_{B G^{I}}^{\otimes 2}$ of the form

$\sum_{I^{\prime} \subset I, J^{\prime} \subset J}(-1)^{\epsilon\left(I^{\prime}, J^{\prime}\right)+\left|\tilde{x}_{\left(J^{\prime}\right)}\right|}\left(\left(1 \otimes \omega_{1} \otimes \tilde{x}_{\left(I^{\prime}\right)}\right) \otimes\left(1 \otimes \omega_{2} \otimes \tilde{x}_{\left(J^{\prime}\right)}\right)\right) \otimes\left(\tilde{x}_{\left(I-I^{\prime}\right)} \otimes \tilde{x}_{\left(J-J^{\prime}\right)}\right)$ with $\epsilon\left(I^{\prime}, J^{\prime}\right)=\epsilon\left(I: I^{\prime}\right)+\epsilon\left(J: J^{\prime}\right)+\left|\tilde{x}_{\left(J^{\prime}\right)}\right|\left|\tilde{x}_{\left(I-I^{\prime}\right)}\right|+\left|\tilde{x}_{\left(J^{\prime}\right)}\right|$. Then

$$
\begin{aligned}
& \operatorname{Dlcop}\left(\left(\omega_{1} \otimes \tilde{x}_{(I)}\right) \otimes\left(\omega_{2} \otimes \tilde{x}_{(J)}\right)\right) \\
= & H(\bar{\varepsilon} \otimes 1) H\left(\Delta^{!} \otimes 1\right) H\left(\bar{\mu}(1 \otimes \bar{T}) \otimes_{\mu^{\prime}} \bar{\zeta}\right)(\theta) \\
= & H(\bar{\varepsilon} \otimes 1) H\left(\Delta^{!} \otimes 1\right) \\
& \quad\left(\sum_{I^{\prime} \subset I, J^{\prime} \subset J}(-1)^{\epsilon\left(I^{\prime} J^{\prime}\right)}\left(1 \otimes \omega_{1} \omega_{2} \otimes \tilde{x}_{\left(I^{\prime}\right)} \tilde{x}_{\left(J^{\prime}\right)}\right) \otimes\left(\tilde{x}_{\left(I-I^{\prime}\right)} \otimes \tilde{x}_{\left(J-J^{\prime}\right)}\right)\right) .
\end{aligned}
$$

If $I \cup J \neq[n]$, then $\left(\Delta^{!} \otimes 1\right)\left(\tilde{x}_{\left(I^{\prime}\right)} \tilde{x}_{\left(J^{\prime}\right)}\right)=0$ for any $I^{\prime} \subset I$ and $J^{\prime} \subset J$ by the definition of $\Delta^{!}$. Thus it is enough to consider the case where $I \cup J=[n]$. Denote by $\epsilon^{\prime}\left(I^{\prime}, J^{\prime}\right)$ the Koszul sign convention satisfying $\tilde{x}_{\left(I^{\prime}\right)} \tilde{x}_{\left(J^{\prime}\right)}=\left(-1 \epsilon^{\epsilon^{\prime}\left(I^{\prime}, J^{\prime}\right)} \tilde{x}_{([n])}\right.$ for $I^{\prime}$ and $J^{\prime}$ with $I^{\prime} \amalg J^{\prime}=[n]$. We have

$$
\begin{aligned}
& \operatorname{Dlcop}\left(\left(\omega_{1} \otimes \tilde{x}_{(I)}\right) \otimes\left(\omega_{2} \otimes \tilde{x}_{(J)}\right)\right) \\
= & H(\bar{\varepsilon} \otimes 1)\left(\sum_{\substack{I^{\prime} \subset I_{,} J^{\prime} \subset J, I^{\prime} \amalg J^{\prime}=[n],}}(-1)^{\epsilon\left(I^{\prime}, J^{\prime}\right)+\epsilon^{\prime}\left(I^{\prime}, J^{\prime}\right)}\left(\omega_{1} \omega_{2} \tilde{x}_{\left(I-I^{\prime}\right)} \otimes \tilde{x}_{\left(J-J^{\prime}\right)}\right)\right) \\
= & (-1)^{\epsilon(I,[n]-I)+\epsilon^{\prime}(I,[n]-I)} \omega_{1} \omega_{2} \tilde{x}_{(J-([n]-I))} \\
= & (-1)^{\epsilon(I, J-I \cap J))+\epsilon^{\prime}(I, J-I \cap J)} \omega_{1} \omega_{2} \tilde{x}_{(I \cap J)} .
\end{aligned}
$$


Since $\epsilon^{\prime}(I, J-I \cap J)=\epsilon_{2}, \epsilon(I: I)=0$ and $\epsilon(J-I \cap J)=\varepsilon_{1}$,

$$
\epsilon(I, J-I \cap J)+\epsilon^{\prime}(I, J-I \cap J)=\epsilon_{1}+\epsilon_{2}+\#(J-I \cap J) .
$$

This completes the proof.

\section{The dual loop (co)product on $H^{*}\left(L\left(E S^{1} \times{ }_{S^{1}} S^{2}\right) ; \mathbb{Q}\right)$}

In this section, we determine the structure of the dual loop (co)product of the Borel construction $E S^{1} \times{ }_{S^{1}} S^{2}$ stated in $\S 1$. Denote by $M=E S^{1} \times{ }_{S^{1}} S^{2}$ in this section. We see that the Borel construction $M$ is a 1-dimensional $\mathbb{Q}$-Gorenstein space by [4, Theorem 4.3] and the fibration $S^{2} \rightarrow M \rightarrow B S^{1}$. Recall that $M$ is homotopy equivalence to the one point union of the infinite dimensional complex projective space $\mathbb{C} P^{\infty}$ and the cohomology ring $H^{*}\left(\mathbb{C} P^{\infty} ; \mathbb{Q}\right)$ is isomorphic to the polynomial algebra $\mathbb{Q}[x]$ with $|x|=2$. Let $\Lambda V$ be a free commutative dg algebra defined by

$$
\Lambda V=(\Lambda(x, y, z), d), d x=d y=0, d z=x y,|x|=|y|=2,|z|=3
$$

Since an algebra map

$$
f: \Lambda V \longrightarrow \mathbb{Q}[x, y] /(x y) \cong H^{*}\left(\mathbb{C} P^{\infty} \vee \mathbb{C} P^{\infty} ; \mathbb{Q}\right) \cong H^{*}(M ; \mathbb{Q})
$$

given by $f(x)=x, f(y)=y, f(z)=0$ is a quasi-isomorphism, $\Lambda V$ is a minimal Sullivan model for $M$. The differential $\bar{D}$ of the model $\mathcal{M}_{L M}=\Lambda(x, y, z) \otimes$ $\Lambda(\tilde{x}, \tilde{y}, \tilde{z})$ stated in $\S 2.1$ satisfies

$$
\bar{D}(\tilde{x})=\bar{D}(\tilde{y})=0, \bar{D}(\tilde{z})=-x \tilde{y}-y \tilde{x}
$$

We first determine a $\mathbb{Q}$-vector space structure of $H^{*}(L M ; \mathbb{Q})$. Recall that the homology decomposes into a direct sum as $\oplus_{i} H_{(i)}^{*}(L M ; \mathbb{Q})$ described in $\S 2.1$.

Lemma 4.1. One has

1. $H_{(0)}^{*}(L M ; \mathbb{Q}) \cong \mathbb{Q}\left\{1, x^{i}, y^{i} \mid i \geq 1\right\}$,

2. $H_{(1)}^{*}(L M ; \mathbb{Q}) \cong \mathbb{Q}\left\{x^{i} \tilde{x}, y^{i} \tilde{y}, x \tilde{y} \mid i \geq 0\right\}$,

3. $H_{(n)}^{*}(L M ; \mathbb{Q}) \cong \mathbb{Q}\left\{\tilde{x} \tilde{y} \tilde{z}^{n-2}, x \tilde{y} \tilde{z}^{n-1}+(n-1) z \tilde{x} \tilde{y} \tilde{z}^{n-2}\right\}(n \geq 2)$

as $\mathbb{Q}$-vector spaces.

Proof. The assertions (1), (2) and (3) are proved by straightforward computations described below. We only show (1) and (2). A similar argument shows the assertion (3). 
(1). By the definition, $H_{(0)}^{*}(L M ; \mathbb{Q}) \cong H^{*}(\Lambda V, d) \cong \mathbb{Q}\left\{1, x^{i}, y^{i} \mid i \geq 1\right\}$.

(2). We can choose a basis for the degree $n$ part $(\Lambda V \otimes(s V))^{n}$ as follows:

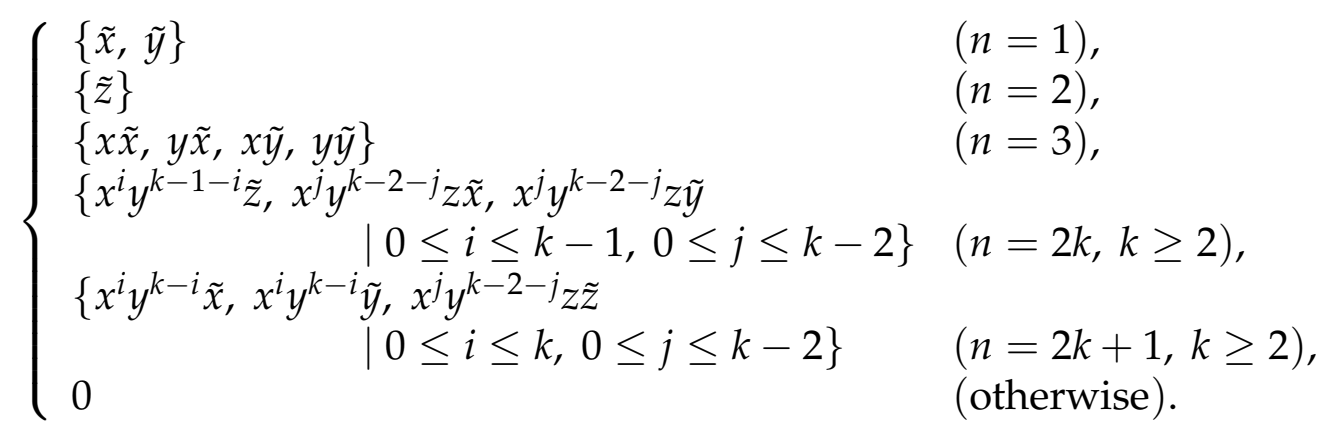

It is easily seen that $H_{(1)}^{1}(L M ; \mathbb{Q}) \cong \mathbb{Q}\{\tilde{x}, \tilde{y}\}$ and $H_{(1)}^{2}(L M ; \mathbb{Q})=0$ since $\tilde{x}$ and $\tilde{y}$ are cycles and $\tilde{z}$ is not a cycle. For $n=3, x \tilde{x}, y \tilde{x}, x \tilde{y}$ and $y \tilde{y}$ are all cycles. Let $r_{i} \in \mathbb{Q}(1 \leq i \leq 4)$. Then, $r_{1} x \tilde{x}+r_{2} y \tilde{x}+r_{3} x \tilde{y}+r_{4} y \tilde{y}$ is a boundary if and only if $r_{1}=r_{4}=0$ and $r_{2}=r_{3}$. We hence have

$$
H_{(1)}^{3}(L M ; \mathbb{Q}) \cong \frac{\left\{r_{1} x \tilde{x}+r_{2} y \tilde{x}+r_{3} x \tilde{y}+r_{4} y \tilde{y} \mid r_{i} \in \mathbb{Q}\right\}}{\mathbb{Q}\{y \tilde{x}+x \tilde{y}\}} \cong \mathbb{Q}\{x \tilde{x}, y \tilde{y}, x \tilde{y}\} .
$$

Consider the case where $n=2 k(k \geq 2)$. For any element $w$ in $(\Lambda V \otimes(s V))^{2 k}$, we may write

$$
w=\sum_{i=0}^{k-1} r_{i} x^{i} y^{k-1-i} \tilde{z}+\sum_{j=0}^{k-2} p_{j} x^{j} y^{k-2-j} z \tilde{x}+\sum_{j=0}^{k-2} q_{j} x^{j} y^{k-2-j} z \tilde{y} .
$$

for some $r_{i}, p_{j}, q_{j} \in \mathbb{Q}$. A straightforward calculation shows that $w$ is a cycle if and only if $r_{0}=r_{k-1}=0, r_{i}=p_{i-1}(1 \leq i \leq k-1)$ and $r_{j}=q_{j}(0 \leq j \leq k-2)$. If $w$ is a cycle, it is readily seen that $w$ is a boundary, that is, $H_{(1)}^{2 k}(L M ; \mathbb{Q})=0$. Similarly, for any $v$ in $(\Lambda V \otimes(s V))^{2 k+1}(k \geq 2)$, we may write

$$
v=\sum_{i=0}^{k} p_{i}^{\prime} x^{i} y^{k-i} \tilde{x}+\sum_{i=0}^{k} q_{i}^{\prime} x^{i} y^{k-i} \tilde{y}+\sum_{j=0}^{k-2} r_{j}^{\prime} x^{j} y^{k-2-j} z \tilde{z}
$$

for some $p_{i}^{\prime}, q_{i}^{\prime}, r_{j}^{\prime} \in \mathbb{Q}$. It is readily seen that $v$ is a cycle if and only if $r_{j}^{\prime}=0$ $(0 \leq j \leq k-2)$. We also see that if $v$ is a cycle, then $v$ is a boundary if and only if $p_{k}^{\prime}=q_{0}^{\prime}=0$. It follows that $H_{(1)}^{2 k+1}(L M ; \mathbb{Q}) \cong \mathbb{Q}\left\{x^{k} \tilde{x}, y^{k} \tilde{y}\right\}$.

For convenience, we put

$$
v_{m}=\tilde{x} \tilde{y} \tilde{z}^{m-2}, w_{n}=x \tilde{y} \tilde{z}^{n-1}+(n-1) z \tilde{x} \tilde{y} \tilde{z}^{n-2}(m \geq 2, n \geq 1)
$$

in $H^{*}\left(\mathcal{M}_{L M}\right)$. Next let us consider the dual loop product and the dual loop coproduct on $H^{*}(L M ; \mathbb{Q})$. The differential $D$ of $\mathcal{M}_{M^{I}}=\Lambda(x, y, z)^{\otimes 2} \otimes \Lambda(\tilde{x}, \tilde{y}, \tilde{z})$ satisfies

$$
\begin{aligned}
& D(\tilde{x})=(-x \otimes 1+1 \otimes x) \otimes 1, D(\tilde{y})=(-y \otimes 1+1 \otimes y) \otimes 1, \\
& D(\tilde{z})=(-z \otimes 1+1 \otimes z) \otimes 1-\frac{1}{2}(x \otimes 1+1 \otimes x) \otimes \tilde{y}-\frac{1}{2}(y \otimes 1+1 \otimes y) \otimes \tilde{x} .
\end{aligned}
$$


For determining the algebraic structures by using the models (2) and (8), it is necessary to find a homology class $\bar{a}$ in $H^{*}\left(\mathcal{M}_{M^{I}} \otimes_{\Lambda V^{\otimes 2}} \mathcal{M}_{M^{I}}\right)$ with $(\bar{\varepsilon} \otimes 1)(\bar{a})=a$ for any element $a$ in $H^{*}\left(\mathcal{M}_{L M}\right)$. The following lemma is needed to give computations of Dlp and Dlcop. Denote by $\left(\begin{array}{l}n \\ i\end{array}\right)=\frac{n !}{i !(n-i) !}$ the binomial coefficients indexed by $n$ and $i$.

Lemma 4.2. Let $\bar{v}_{m}$ and $\bar{w}_{n}$ be elements in $\mathcal{M}_{M^{I}} \otimes_{\Lambda V^{\otimes 2}} \mathcal{M}_{M^{I}}$ given by

$$
\begin{aligned}
& \bar{v}_{m}= \sum_{i=0}^{m-2}\left(\begin{array}{c}
m-2 \\
i
\end{array}\right)\left(\tilde{z}^{i} \otimes \tilde{x} \tilde{y} \tilde{z}^{m-2-i}+\tilde{x} \tilde{z} \otimes \tilde{y} \tilde{z}^{m-2-i}\right. \\
&\left.-\tilde{y} \tilde{z}^{i} \otimes \tilde{x} \tilde{z}^{m-2-i}+\tilde{x} \tilde{y} \tilde{z}^{i} \otimes \tilde{z}^{m-2-i}\right) \\
& \bar{w}_{n}= \sum_{i=0}^{n-2} \frac{(n-1) !}{i !(n-2-i) !}\left(\left(1 \otimes z \otimes \tilde{z}^{i}\right) \otimes \tilde{x} \tilde{y} \tilde{z}^{n-2-i}+\left(1 \otimes z \otimes \tilde{x} \tilde{z}^{i}\right) \otimes \tilde{y} \tilde{z}^{n-2-i}\right. \\
&\left.\quad-\left(1 \otimes z \otimes \tilde{y} \tilde{z}^{i}\right) \otimes \tilde{x} \tilde{z}^{n-2-i}+\left(1 \otimes z \otimes \tilde{x} \tilde{y} \tilde{z}^{i}\right) \otimes \tilde{z}^{n-2-i}\right) \\
&+\sum_{i=0}^{n-1}\left(\begin{array}{c}
n-1 \\
i
\end{array}\right)\left(\left(1 \otimes x \otimes \tilde{z}^{i}\right) \otimes \tilde{y} \tilde{z}^{n-1-i}+\left(1 \otimes x \otimes \tilde{y} \tilde{z}^{i}\right) \otimes \tilde{z}^{n-1-i}\right) \\
&+\frac{1}{2} \sum_{i=0}^{n-2} \frac{(n-1) !}{i !(n-2-i) !}\left(-\left(1 \otimes x \otimes \tilde{y} \tilde{z}^{i}\right) \otimes \tilde{x} \tilde{y} \tilde{z}^{n-2-i}\right. \\
&\left.\quad+\left(1 \otimes x \otimes \tilde{x} \tilde{y} \tilde{z}^{i}\right) \otimes \tilde{y} \tilde{z}^{n-2-i}\right) .
\end{aligned}
$$

Then $\bar{v}_{m}$ and $\bar{w}_{n}$ are cycles and the map $\bar{\varepsilon} \otimes 1$ sends $\bar{v}_{m}$ and $\bar{w}_{n}$ to $v_{m}$ and $w_{n}$, respectively. Proof. It is easily seen that $(\bar{\varepsilon} \otimes 1)\left(\bar{v}_{m}\right)=v_{m}$ and $(\bar{\varepsilon} \otimes 1)\left(\bar{w}_{n}\right)=w_{n}$. Moreover, we see that $\bar{v}_{m}$ and $\bar{w}_{n}$ are cycles by straightforward computations.

We next construct a generator of $\operatorname{Ext}_{\Lambda V^{\otimes 2}}^{1}\left(\Lambda V, \Lambda V^{\otimes 2}\right)$. Define a right $\Lambda V^{\otimes 2}$ module map $\Delta^{!}: \mathcal{M}_{M^{I}} \rightarrow \Lambda V^{\otimes 2}$ by

$$
\begin{aligned}
& \Delta^{!}(\tilde{x} \tilde{y})=-z \otimes 1+1 \otimes z, \\
& \Delta^{!}(\tilde{x})=\frac{1}{2}(x \otimes 1+1 \otimes x), \Delta^{!}(\tilde{y})=-\frac{1}{2}(y \otimes 1+1 \otimes y)
\end{aligned}
$$

and $\Delta^{!}\left(\tilde{z}^{i}\right), \Delta^{!}\left(\tilde{x} \tilde{z}^{j}\right), \Delta^{!}\left(\tilde{y} \tilde{z}^{j}\right), \Delta^{!}\left(\tilde{x} \tilde{y} \tilde{z}^{j}\right)(i \geq 0, j \geq 1)$ are all zero. Then, $\Delta^{!}$is a chain map of degree 1 and a generator of

$$
\operatorname{Ext}_{\Lambda V^{\otimes 2}}^{1}\left(\Lambda V, \Lambda V^{\otimes 2}\right) \cong \operatorname{Ext}_{C^{*}\left(M^{2}\right)}^{1}\left(C^{*}(M), C^{*}\left(M^{2}\right)\right) \cong \mathbb{Q} .
$$

By using the model (2), we give a computation of the dual loop product as follows.

Theorem 4.3. One has $\operatorname{Dlp}\left(H_{(j)}^{*}(L M ; \mathbb{Q})\right)=\{0\}(j=0,1)$ and

$$
\begin{aligned}
& \operatorname{Dlp}\left(v_{2}\right)=0 \\
& \operatorname{Dlp}\left(v_{m}\right)=\sum_{i=1}^{m-2} \frac{(m-2) !}{(i-1) !(m-2-i) !}\left(w_{i} \otimes v_{m-i}-v_{i+1} \otimes w_{m+1-i}\right)(m \geq 3), \\
& \operatorname{Dlp}\left(w_{n}\right)=\sum_{i=1}^{n-1} \frac{(n-1) !}{(i-1) !(n-1-i) !} w_{i} \otimes w_{n-i} .
\end{aligned}
$$


Proof. Recall the model for the dual loop product (2). Since $\Delta^{!}(1)=0$, $\operatorname{Dlp}\left(H_{(j)}^{*}(L M ; \mathbb{Q})\right)=\{0\}$ for $j=0,1$. Indeed,

$$
\begin{aligned}
\operatorname{Dlp}\left(x^{i} \tilde{x}\right)= & H\left(\Delta^{!} \otimes 1\right) \circ H(\bar{\varepsilon} \otimes 1)^{-1} \circ H\left((\mu \otimes 1) \otimes_{\mu}(\mu \otimes 1)\right) \\
& \left(\left(1 \otimes x^{i} \otimes \tilde{x}\right) \otimes 1+\left(1 \otimes x^{i} \otimes 1\right) \otimes \tilde{x}\right) \\
= & H\left(\Delta^{!} \otimes 1\right) \circ H(\bar{\varepsilon} \otimes 1)^{-1}\left(x^{i} \otimes(\tilde{x} \otimes 1+1 \otimes \tilde{x})\right) \\
= & H\left(\Delta^{!} \otimes 1\right)\left(\left(1 \otimes x^{i} \otimes 1\right) \otimes(\tilde{x} \otimes 1+1 \otimes \tilde{x})\right)=0 .
\end{aligned}
$$

A similar argument shows that $\operatorname{Dlp}(1), \operatorname{Dlp}\left(x^{i}\right), \operatorname{Dlp}\left(y^{i}\right), \operatorname{Dlp}\left(y^{i} \tilde{y}\right)$ and $\operatorname{Dlp}(x \tilde{y})$ are all zero.

Next let us compute $\operatorname{Dlp}\left(v_{m}\right)$. Let $\bar{v}_{m}$ be a cycle of $\mathcal{M}_{M^{I}} \otimes_{\Lambda V^{\otimes 2}} \mathcal{M}_{M^{I}}$ described in Lemma 4.2. Then

$$
\operatorname{Dlp}\left(v_{m}\right)=H\left(\Delta^{!} \otimes 1\right) H(\bar{\varepsilon} \otimes 1)^{-1} H\left((\mu \otimes 1) \otimes_{\mu}(\mu \otimes 1)\right)\left(\bar{v}_{m}\right) .
$$

We so need to find a cycle $\bar{v}_{m}^{\prime}$ in $\mathcal{M}_{M^{I}} \otimes_{\Lambda V^{\otimes 2}}\left(\mathcal{M}_{L M} \otimes \mathcal{M}_{L M}\right)$ with $H(\bar{\varepsilon} \otimes 1)\left(\bar{v}_{m}^{\prime}\right)=$ $H\left((\mu \otimes 1) \otimes_{\mu}(\mu \otimes 1)\right)\left(\bar{v}_{m}\right)$. Denote by $\bar{v}_{m}^{\prime}$ a element of $\mathcal{M}_{M^{I}} \otimes_{\Lambda V^{\otimes 2}}$ $\left(\mathcal{M}_{L M} \otimes \mathcal{M}_{L M}\right)$ given by

$$
\begin{aligned}
\sum_{i=0}^{m}( & \left(\begin{array}{c}
m-2 \\
i
\end{array}\right) 1 \otimes\left(\tilde{z}^{i} \otimes \tilde{x} \tilde{y} \tilde{z}^{m-2-i}+\left(\begin{array}{c}
m-2 \\
i-1
\end{array}\right) 1 \otimes\left(\tilde{x} \tilde{z}^{i-1} \otimes \tilde{y} \tilde{z}^{m-1-i}\right)\right. \\
& -\left(\begin{array}{c}
m-2 \\
i-1
\end{array}\right) 1 \otimes\left(\tilde{y} \tilde{z}^{i-1} \otimes \tilde{x} \tilde{z} \tilde{z}^{m-1-i}\right)+\left(\begin{array}{c}
m-2 \\
i-2
\end{array}\right) 1 \otimes\left(\tilde{x} \tilde{z} \tilde{z}^{i-2} \otimes \tilde{z}^{m-i}\right) \\
& +i\left(\begin{array}{c}
m-2 \\
i
\end{array}\right)\left(\tilde{x} \otimes\left(\tilde{y} \tilde{z}^{i-1} \otimes \tilde{x} \tilde{y} \tilde{z}^{m-2-i}\right)+\tilde{y} \otimes\left(\tilde{x} \tilde{z}^{i-1} \otimes \tilde{x} \tilde{y} \tilde{z}^{m-2-i}\right)\right) \\
& -(m-i)\left(\begin{array}{c}
m-2 \\
i-2
\end{array}\right)\left(\tilde{x} \otimes\left(\tilde{x} \tilde{y} \tilde{z}^{i-2} \otimes \tilde{y} \tilde{z}^{m-1-i}+\tilde{y} \otimes\left(\tilde{x} \tilde{y} \tilde{z}^{i-2} \otimes \tilde{x} \tilde{z}^{m-1-i}\right)\right)\right. \\
& \left.+i(i-1)\left(\begin{array}{c}
m-2 \\
i
\end{array}\right) \tilde{x} \tilde{y} \otimes\left(\tilde{x} \tilde{y} \tilde{z}^{i-2} \otimes \tilde{x} \tilde{y} \tilde{z}^{m-2-i}\right)\right) .
\end{aligned}
$$

Here we put $\left(\begin{array}{l}n \\ j\end{array}\right)=0$ where $j<0$ or $j>n$ for convenience. A straightforward calculation shows that $\bar{v}_{m}^{\prime}$ is a cycle and the equation

$$
H(\bar{\varepsilon} \otimes 1)\left(\bar{v}_{m}^{\prime}\right)=H\left((\mu \otimes 1) \otimes_{\mu}(\mu \otimes 1)\right)\left(\bar{v}_{m}\right)
$$

holds. In the homology $H^{*}\left(\mathcal{M}_{L M}\right)$, we have

$$
w_{i}=\frac{1}{2} x \tilde{y} \tilde{z}^{i-1}-\frac{1}{2} y \tilde{x} \tilde{z}^{i-1}+(i-1) z \tilde{x} \tilde{y} \tilde{z}^{i-2}
$$

for $i \geq 2$. Moreover the following equations hold in $H^{*}\left(\mathcal{M}_{L M}^{\otimes 2}\right)$ :

$$
\begin{aligned}
& (m-1-i) \tilde{x} \tilde{z}^{i-1} \otimes y \tilde{x} \tilde{y} \tilde{z}^{m-2-i}+(i-1) x \tilde{x} \tilde{y} \tilde{z}^{i-2} \otimes \tilde{y} \tilde{z} \tilde{z}^{m-1-i}=0, \\
& (m-1-i) \tilde{y} \tilde{z}^{i-1} \otimes x \tilde{x} \tilde{y} \tilde{z}^{m-2-i}+(i-1) y \tilde{x} \tilde{y} \tilde{z}^{i-2} \otimes \tilde{x} \tilde{z}^{m-1-i}=0 .
\end{aligned}
$$


By these equations, we conclude that $\operatorname{Dlp}\left(v_{2}\right)=H\left(\Delta^{!} \otimes 1\right)\left(\bar{v}_{2}^{\prime}\right)=0$ and

$$
\begin{aligned}
\operatorname{Dlp}\left(v_{m}\right) & =H\left(\Delta^{!} \otimes 1\right)\left(\bar{v}_{m}^{\prime}\right) \\
& =\sum_{i=1}^{m-2} \frac{(m-2) !}{(i-1) !(m-2-i) !}\left(w_{i} \otimes v_{m-i}-v_{i+1} \otimes w_{m+1-i}\right)
\end{aligned}
$$

for $m \geq 3$. Similarly, we can compute $\operatorname{Dlp}\left(w_{n}\right)$ as below. Let $\bar{w}_{n}$ be a cycle in $\mathcal{M}_{M^{I}} \otimes_{\Lambda V^{\otimes 2}} \mathcal{M}_{M^{I}}$ described in Lemma 4.2. Denote by $\bar{w}_{n+1}^{\prime}$ a element of $\mathcal{M}_{M^{I}} \otimes_{\Lambda V \otimes 2}\left(\mathcal{M}_{L M} \otimes \mathcal{M}_{L M}\right)$ given by

$$
\begin{aligned}
& \sum_{i=0}^{n+1}\left(n\left(\begin{array}{c}
n-1 \\
i
\end{array}\right) 1 \otimes\left(z \tilde{z}^{i} \otimes \tilde{x} \tilde{y} \tilde{z}^{n-1-i}\right)+n\left(\begin{array}{c}
n-1 \\
i-2
\end{array}\right) 1 \otimes\left(z \tilde{x} \tilde{y} \tilde{z}^{i-2} \otimes \tilde{z}^{n+1-i}\right)\right. \\
& +\left(\begin{array}{c}
n \\
i
\end{array}\right) 1 \otimes\left(x \tilde{z}^{i} \otimes \tilde{y} \tilde{z}^{n-i}\right)+\left(\begin{array}{c}
n \\
i-1
\end{array}\right) 1 \otimes\left(x \tilde{y} \tilde{z}^{i-1} \otimes \tilde{z}^{n+1-i}\right) \\
& +n\left(\begin{array}{c}
n-1 \\
i-1
\end{array}\right) 1 \otimes\left(z \tilde{x} \tilde{z}^{i-1} \otimes \tilde{y} \tilde{z}^{n-i}\right)-n\left(\begin{array}{c}
n-1 \\
i-1
\end{array}\right) 1 \otimes\left(z \tilde{y} \tilde{z}^{i-1} \otimes \tilde{x} \tilde{z}^{n-i}\right) \\
& -n\left(\begin{array}{c}
n-1 \\
i
\end{array}\right) \tilde{y} \otimes\left(x \tilde{z}^{i} \otimes \tilde{x} \tilde{y} \tilde{z}^{n-1-i}\right) \\
& +(n+1-i)\left(\begin{array}{c}
n \\
i-1
\end{array}\right)\left(\tilde{x} \otimes\left(x \tilde{y} \tilde{z}^{i-1} \otimes \tilde{y} \tilde{z}^{n-i}\right)+\tilde{y} \otimes\left(x \tilde{y} \tilde{z}^{i-1} \otimes \tilde{x} \tilde{z}^{n-i}\right)\right) \\
& -i n\left(\begin{array}{c}
n-1 \\
i
\end{array}\right)\left(\tilde{x} \otimes\left(z \tilde{y} \tilde{z}^{i-1} \otimes \tilde{x} \tilde{y} \tilde{z}^{n-1-i}\right)+\tilde{y} \otimes\left(z \tilde{x} \tilde{z}^{i-1} \otimes \tilde{x} \tilde{y} \tilde{z}^{n-1-i}\right)\right) \\
& +n(n+1-i)\left(\begin{array}{c}
n-1 \\
i-2
\end{array}\right)\left(\tilde{x} \otimes\left(z \tilde{x} \tilde{y} \tilde{z}^{i-2} \otimes \tilde{y} \tilde{z}^{n-i}\right)\right. \\
& \left.\left.+\tilde{y} \otimes\left(z \tilde{x} \tilde{y} \tilde{z}^{i-2} \otimes \tilde{x} \tilde{z}^{n-i}\right)\right)\right) \\
& -\sum_{i=0}^{n} \frac{n}{2}\left(\left(\begin{array}{c}
n-1 \\
i
\end{array}\right) 1 \otimes\left(x \tilde{y} \tilde{z}^{i} \otimes \tilde{x} \tilde{y} \tilde{z}^{n-1-i}\right)-\left(\begin{array}{c}
n-1 \\
i-1
\end{array}\right) 1 \otimes\left(x \tilde{x} \tilde{y} \tilde{z}^{i-1} \otimes \tilde{y} \tilde{z}^{n-i}\right)\right. \\
& \left.+i\left(\begin{array}{c}
n-1 \\
i
\end{array}\right) \tilde{y} \otimes\left(x \tilde{x} \tilde{y} \tilde{z}^{i-1} \otimes \tilde{x} \tilde{y} \tilde{z}^{n-1-i}\right)\right) .
\end{aligned}
$$

It is readily seen that the element $\bar{w}_{n}^{\prime}$ is a cycle and $H(\bar{\varepsilon} \otimes 1)\left(\bar{w}_{n}^{\prime}\right)=H\left((\mu \otimes 1) \otimes_{\mu}\right.$ $(\mu \otimes 1))\left(\bar{w}_{n}\right)$. Therefore, by a straightforward calculation,

$$
\operatorname{Dlp}\left(w_{n}\right)=H\left(\Delta^{!} \otimes 1\right)\left(\overline{w_{n}^{\prime}}\right)=\sum_{i=1}^{n-1} \frac{(n-1) !}{(i-1) !(n-1-i) !} w_{i} \otimes w_{n-i} .
$$

This completes the proof.

In the rest of this paper, we give a computation of the dual loop coproduct on $H^{*}(L M ; \mathbb{Q})$. Let $\bar{T}: \mathcal{M}_{M^{I}} \rightarrow \mathcal{M}_{M^{I}}$ be a map defined by $\bar{T}(a \otimes b \otimes 1)=$ $(-1)^{|a||b|} b \otimes a \otimes 1, \bar{T}(\tilde{x})=-\tilde{x}, \bar{T}(\tilde{y})=-\tilde{y}$ and $\bar{T}(\tilde{z})=-\tilde{z}$ for $a, b \in \Lambda V$. This is 
just a map described in the diagram (6). We also see that $-\Delta^{!} \bar{T}=T \Delta^{!}$. By Lemma 2.3 , it implies that

$$
\operatorname{Dlcop}\left(a_{1} \otimes a_{2}\right)=(-1)^{\left|a_{1}\right|\left|a_{2}\right|+1} \operatorname{Dlcop}\left(a_{2} \otimes a_{1}\right)
$$

for $a_{1}, a_{2} \in H^{*}(L M ; \mathbb{Q})$.

Theorem 4.4. One has

$$
\begin{aligned}
& \operatorname{Dlcop}\left(H^{*}(L M ; \mathbb{Q}) \otimes H_{(\geq 2)}^{*}(L M ; \mathbb{Q})\right)=\{0\}, \\
& \operatorname{Dlcop}\left(H_{(\geq 2)}^{*}(L M ; \mathbb{Q}) \otimes H^{*}(L M ; \mathbb{Q})\right)=\{0\},
\end{aligned}
$$

and for generators $a, b$ in $H_{(\leq 1)}^{*}(L M ; Q)$ described in Theorem 4.1, the following equations hold;

$$
\operatorname{Dlcop}(a \otimes b)= \begin{cases}-x^{i+j+1} & (a, b)=\left(x^{i}, x^{j} \tilde{x}\right), \\ x^{i+j+1} \tilde{x} & (a, b)=\left(x^{i} \tilde{x}, x^{j} \tilde{x}\right) \\ y^{i+j+1} & (a, b)=\left(y^{i}, y^{j} \tilde{y}\right), \\ -y^{i+j+1} \tilde{y} & (a, b)=\left(y^{i} \tilde{y}, y^{j} \tilde{y}\right), \\ x \tilde{y} & (a, b)=(\tilde{x}, \tilde{y}), \\ 0 & \text { (otherwise). }\end{cases}
$$

Proof. It is easily seen that these elements

$$
\begin{aligned}
& \left(1 \otimes x^{i} \otimes 1\right) \otimes 1, \quad\left(1 \otimes y^{i} \otimes 1\right) \otimes 1, \\
& \left(1 \otimes x^{i} \otimes \tilde{x}\right) \otimes 1+\left(1 \otimes x^{i} \otimes 1\right) \otimes(1 \otimes 1 \otimes \tilde{x}), \\
& \left(1 \otimes y^{i} \otimes \tilde{y}\right) \otimes 1+\left(1 \otimes y^{i} \otimes 1\right) \otimes(1 \otimes 1 \otimes \tilde{y}),
\end{aligned}
$$

are cycles in $\mathcal{M}_{M^{I}} \otimes_{\Lambda V^{\otimes 2}} \mathcal{M}_{M^{I}}$ and the map $\bar{\varepsilon} \otimes 1$ sends each cycles to $x^{i}, y^{i}, x^{i} \tilde{x}$ and $y^{i} \tilde{y}$, respectively. Hence, by a straightforward computation,

$$
\begin{aligned}
& \operatorname{Dlcop}\left(x^{j} \otimes x^{i} \tilde{x}\right)=-\operatorname{Dlcop}\left(x^{i} \tilde{x} \otimes x^{j}\right)=-x^{i+j+1}, \\
& \operatorname{Dlcop}\left(x^{i} \tilde{x} \otimes x^{j} \tilde{x}\right)=x^{i+j+1} \tilde{x}, \\
& \operatorname{Dlcop}\left(y^{j} \otimes y^{i} \tilde{y}\right)=-\operatorname{Dlcop}\left(y^{i} \tilde{y} \otimes y^{j}\right)=y^{i+j+1}, \\
& \operatorname{Dlcop}\left(y^{i} \tilde{y} \otimes y^{j} \tilde{y}\right)=-y^{i+j+1} \tilde{y}, \\
& \operatorname{Dlcop}(\tilde{x} \otimes \tilde{y})=\operatorname{Dlcop}(\tilde{y} \otimes \tilde{x})=x \tilde{y} .
\end{aligned}
$$

Let $\bar{v}_{m}$ and $\bar{w}_{n}$ be cycles in $\mathcal{M}_{M^{I}} \otimes_{\Lambda V^{\otimes 2}} \mathcal{M}_{M^{I}}$ described in Lemma 4.2. We may write $\bar{v}_{m}=\sum \bar{v}_{m(1)} \otimes \bar{v}_{m(2)}$ and $\bar{w}_{n}=\sum \bar{w}_{n(1)} \otimes \bar{w}_{n(2)}$ for some $\bar{v}_{m(1)}, \bar{v}_{m(2)}, \bar{w}_{n(1)}$, $\bar{w}_{n(2)}$ in $\mathcal{M}_{M^{I}}$. By the definition of $\bar{v}_{m}$, we have $\Delta^{!}\left(\bar{v}_{m(1)}\right)=0$ or $(\bar{\varepsilon} \otimes 1)\left(\bar{v}_{m(2)}\right)=$ 0 . It implies that

$$
\operatorname{Dlcop}\left(\alpha \otimes v_{m}\right)=-\operatorname{Dlcop}\left(v_{m} \otimes \alpha\right)=0
$$

for any $\alpha \in H^{*}(L M ; \mathbb{Q})$. Similarly, at least one of $\Delta^{!}\left(\bar{w}_{n(1)}\right)$ and $(\bar{\varepsilon} \otimes 1)\left(\bar{w}_{n(2)}\right)$ are zero. It follows that $\operatorname{Dlcop}\left(\alpha \otimes w_{n}\right)=-\operatorname{Dlcop}\left(w_{n} \otimes \alpha\right)=0$. 


\section{References}

[1] M. Chas and D. Sullivan, String topology, preprint (1999), arXiv:math.GT/9911159.

[2] R. L. Cohen, V. Godin, A polarized view of string topology, Topology, geometry and quantum field theory, 127-154, London Math. Soc. Lecture Note Ser., 308, Cambridge Univ. Press, Cambridge, 2004.

[3] R. L. Cohen, J. D. S. Jones, J. Yan, The loop homology algebra of spheres and projective spaces. Categorical decomposition techniques in algebraic topology (Isle of Skye, 2001), 77-92, Progr. Math., 215, Birkhauser, Basel, 2004.

[4] Y. Félix, S. Halperin, J. -C. Thomas, Gorenstein spaces. Adv. in Math. 71 (1988), no. $1,92-112$.

[5] Y. Félix, S. Halperin, J. -C. Thomas, Rational Homotopy Theory, Graduate Texts in Mathematics, 205. Springer-Verlag.

[6] Y. Félix and J. -C. Thomas, String topology on Gorenstein spaces, Math. Ann., 345 (2009), no. 2, 417-452.

[7] R. Hepworth, String topology for Lie groups, J. Topol. 3 (2010), no 2, 424-442.

[8] K. Kuribayashi and L. Menichi, On the loop (co)products on the classifying space of a Lie group, in preparation.

[9] A. P. M. Kupers, An elementary proof of the string topology structure of compact oriented surface, preprint (2012), arXiv:math/1110.1158v2.

[10] L. Smith, Homological algebra and the Eilenberg-Moore spectral sequence. Trans. Amer. Math. Soc. 129 (1967) 58-93.

[11] H. Tamanoi, Loop coproducts in string topology and triviality of higher genus TQFT operations. J. Pure Appl. Algebra 214 (2010), no. 5, 605-615.

[12] D. Vaintrob, The string topology BV algebra, Hochschild cohomology and the Goldman bracket on surfaces, preprint(2007), arXiv:math/0702859v1.

[13] M. Vigué-Poirrier, Décompositions de l'homologie cyclique des algèbres différentielles graduées commutatives. (French) [Decomposition of the cyclic homology of commutative graded differential algebras] K-Theory 4 (1991), no. 5, 399-410.

Department of Mathematical Sciences, University of Tokyo,

3-8-1 Komaba Meguro-ku

Tokyo 153-8914 Japan

email:tnaito@ms.u-tokyo.ac.jp 